\title{
Shadow Modelling Based Upon Rayleigh Scattering and
}

\section{Mie Theory}

\author{
Lin $\mathrm{Gu}^{1 *}$ and Antonio Robles-Kelly ${ }^{1,2}$ \\ ${ }^{1}$ School of Engineering, Australian National University, Canberra ACT 0200, Australia \\ ${ }^{2}$ National ICT Australia (NICTA) ${ }^{\dagger}$ Locked Bag 8001, Canberra ACT 2601, Australia
}

\begin{abstract}
In this paper, we present a method to model shadows in outdoor scenes. Here, we note that the shadow areas correspond to the diffuse skylight which arises from the scattering of the sunlight by particles in the atmosphere. This yields a treatment in which shadows in the image can be viewed as a linear combination of scattered light obeying Rayleigh scattering and Mie theory. This allows for the computation of a ratio which permits casting the problem of recovering the shadowed areas in the image into a clustering setting making use of active contours. This also opens-up the formulation of a metric that can be used to assess the degree upon which the scene is overcast. We illustrate the utility of the method for purposes of detecting shadows in real-world imagery, provide time complexity results and compare against a number of alternatives elsewhere in the literature.
\end{abstract}

Keywords: Shadow detection, shadow removal, light scattering

\footnotetext{
${ }^{*}$ Corresponding author. E-mail: lin.gu@ @icta.com.au Tel: +61(2)6267-6285

${ }^{\dagger}$ NICTA is funded by the Australian Government as represented by the Department of Broadband, Communications and the Digital Economy and the Australian Research Council through the ICT Centre of Excellence program.
} 


\section{Introduction}

Shadow detection and removal is an important preprocessing step for purposes of object recognition, video surveillance and segmentation [32]. This is particularly relevant in outdoor environments, where strong shadows ensue due to overcast conditions in addition to cast and selfshadowing. Despite recent interest, shadow detection in outdoor scenes remains a challenging task. Existing methods are often restricted to indoor environments [33], require prior knowledge regarding the illumination setting and geometry [17], employ multiple images [10] or require user input [34].

Indeed, a wide variety of techniques have been proposed, which employ a wide range of features and models to detect and remove cast shadows. These include chromaticity $[8,29]$, scene or object geometry $[9,15]$ and texture [30,21]. Along these lines, Cucchiara et al. [8] use the HSV colour space base upon the intuition that such colour space provides a natural separation between the chromaticity and the luminosity. Salvador et al. [29], in the other hand, use the c1c2c3 colour space, i.e. the hexadecimal RGB colour triplet, over an image region so as to reduce the effects of noise corruption.

In [29], the authors also employ the geometrical properties of the shadows. They do this by following Funka-Lea and Bajcsy [12], who present a number of low computational cost cues for shadow recognition. Fang et al. [9] employ a geometry model, whereby they assume the scene background to be a flat surface. In an alternative approach, Hsieh et al. [15] separate the scenes into blobs so as to recover individual objects for purposes of geometric analysis. Their method hinges in the notion that, as the blobs corresponding to objects in the scene may have different orientations, the extreme points in the blobs can be used to recover shadow-object pairs based upon a Gaussian model defined in terms of the pixel coordinates and their intensities.

As mentioned earlier, textures have also been used for shadow detection. This hinges in the rationale that texture correlation is expected to be invariant to illumination changes and, hence, 
robust to shadowing. This is exploited by Leone and Distante [21], who describe textural information in terms of redundant systems of functions so as to improve the background model used for shadow detection. Sanin et al. [30] use gradient-based texture correlation to discriminate amongst candidate shadow regions.

Alternative approaches include shadow flows [26], i.e. a disparity vector computed by comparing a shadow model with a background model, multiple views and the use of user input. This is the case for the work presented in [10], where the authors recover a shadow-free image based upon the assumption that the illumination varies slowly and, hence, gives rise to the small gradients in each view. As a result, large gradients depend on reflectance changes. The shadow-free image is then compared with the input image so as to recover the shadow edges via thresholding.

As related to the use of user input, in [34], a probabilistic method and matting are used to remove shadows based upon a quadmap [35]. The application of statistics is somewhat related to the method in [33], where a dynamic conditional Markov random field is used to detect shadows and perform background subtraction in indoor scenes. In [22], the authors employ a Gaussian mixture model to characterise the moving cast shadows on the surfaces across the scene.

In this paper, we tackle the problem of detecting shadows in outdoor environments by viewing the shadowed areas in the image as being lit by skylight, whereas the non-shadowed regions in the image are illuminated by both, skylight and sunlight. This is akin to the treatment presented in [13], where the authors detect shadows using Rayleigh and Mie scattering theory. Following [13], we note that the edges in the image produced by shadows should correspond to a mix of sunlight to skylight whose ratio is constant throughout the scene.

This paper is, hence, a natural extension of [13] with a number of major improvements. Firstly, spherical harmonics are used so as to achieve the relaxation of the single point light source assumption in [13]. This is an important theoretical development since it assures our method holds for complex sky luminance patterns, where the direction of the skylight that illuminates the area of 
interest can depart greatly from a single point light source assumption. This is as light scattered by cloud and haze can follow multiple paths, impinging the object surface from arbitrary directions. We use these spherical harmonics so as to cast the problem into a segmentation setting where techniques such as active contours [2] can be used to detect the shadows in the image. This yields a result in accordance with that in [13], where the active contour evolution is governed by a ratio that arises from the use of Rayleigh scattering and Mie theory to model the skylight illuminating the shadowed areas.

Secondly, here we note that, in practice, the shadow area is not always distinct from sunlit area. This is particularly true for the fringe of the shadows in the image, where the transition region, i.e. penumbra, consists of sunlight and skylight mixed with one another. Thus, here, instead of segmenting the image in a binary setting, we recover the proportion of sunlight to skylight along the penumbra area. We illustrate how recovering the exact proportion of these two components can be tackled using image matting by post-processing the shadow boundary with KNN Matting [6].

Thirdly, we propose a weather metric so as to estimate meteorological conditions from single images. Our metric contrasts in simplicity with other methods elsewhere in the literature which aim at inferring atmospheric phenomena for the sky appearance. For instance, the International Commission on Illumination (CIE) has established a standard sky model [7] with five degrees of freedom. This model, proposed by Preetham, Shirly and Smits [27], depends upon five parameters which account for different weather conditions and climates. Despite effective, the model's complexity makes it cumbersome to use in practical settings. As a result, researchers have attempted to use a single parameter, i.e. turbidity, to describe the effect of Mie scattering effect. We remit the interested reader to the detailed description in the classic book by Minnaert [23].

It is also worth noting that here we use a wavelength dependent Mie scattering term. This contrasts with the one used in [13], where a simplified expression devoid of wavelength dependence was used. Thus, the method described here relies on the estimation of model parameters before 
shadow detection. We tackle this drawback by applying an initialisation step which estimates the initial model parameters from the input image. The initialisation presented here is robust to various weather conditions.

The paper is organised as follows. In the following section, we model the skylight as a linear combination of the Rayleigh and Mie scattered light. With this linear combination at hand, we then present the ratio used for the evolution of the active contour, which we present in Section 4. From the ratio, we propose a weather metric to estimate the meteorological condition of clouds obscuring the sky. This is, we propose a method to appraise the degree upon which the sky is overcast based upon a single parameter. We elaborate on the implementation of our method in Section 5. Finally, we present results and conclusions in Sections 6 and 7, respectively.

\section{Rayleigh Scattering and Mie Theory}

As mentioned earlier, here, we note that, in outdoor scenes, the shadowed areas correspond to the diffuse skylight which arises from the scattering of the sunlight by particles in the atmosphere [24]. Thus, we employ the Rayleigh scattering and Mie theory of sunlight propagation in the atmosphere to model the shadows.

Recall that, when sunlight enters the atmosphere, it is scattered by the particles in the air. When these particles are small as compared to the wavelength of the impinging light (typically less than 1 tenth the wavelength), the scattering can be approximated by the proportion of the fourth power of the wavelength of the sun light, i.e. the Rayleigh scattering [16]. It is worth noting in passing that this provides a physical explanation for the sky being blue, as the blue light in shorter in wavelength and, hence, is scattered much more than the red light corresponding to longer wavelengths. The Rayleigh scattering is given by

$$
E_{\text {Rayleigh }}=\frac{8 \pi^{3}\left(r^{2}-1\right)^{2}}{3 N \lambda^{4}}\left(\frac{6+3 p_{n}}{6-7 p_{n}}\right) E(\lambda)
$$


where $E(\lambda)$ is the power spectrum of the illuminant at wavelength $\lambda, r=1.0003$ is the refractive index of air in the visible spectrum, $N=2.545 \times 10^{25}$ is number of molecules per unit volume and $p_{n}$ is the depolarization factor, which is considered to be 0.035 for air.

However, when the sunlight is scattered by particles bigger or of equal size to the wavelength, the scattering phenomenon is modelled by Mie theory [16]. Mie theory states that the scattering is proportional to the second power of the wavelength. Mie theory is generally employed to model the scattering caused by haze in the atmosphere. This is as light scatters more uniformly across wavelengths, which causes a whitewash appearance in haze and cloud. Mie scattering is given by

$$
E_{\text {Mie }}=0.434 B c \pi\left(\frac{2 \pi}{\lambda}\right)^{v-2} E(\lambda)
$$

where $c$ is the concentration factor that varies with turbidity $T$ in the inteval $(0.6544 T-0.6510) \times$ $10^{(-16)}, v$ is Junge's exponent with a value of 4 for the sky and $B=0.68$ in the visible spectrum.

Thus, both, Mie theory and Rayleigh scattering must be taken into account for modelling the skylight. This is as the air in the atmosphere will account for a large fraction of the Rayleght scattering whereas Mie theory is bound to apply to clouds and dust. Both the Rayleigh and Mie scattered light compose the skylight and, hence, we can write

$$
E_{\text {sky }}(\lambda)=\left(1-p_{c}\right) E_{\text {rayleigh }}(\lambda)+p_{c} E_{\text {mie }}(\lambda)
$$

where, $\lambda$ is the wavelength parameter, $p_{c}$ is the contribution of the Mie scattering and $E_{\text {mie }}(\lambda)$ and $E_{\text {rayleigh }}(\lambda)$ corresponds to the Rayleigh scattered light. This equation would be further simplified as

$$
E_{\text {sky }}(\lambda)=\left(\frac{T_{1}}{\lambda^{4}}+\frac{T_{2}}{\lambda^{2}}\right) E_{\text {sun }}
$$

Here we use two term $T_{1}$ and $T_{2}$ to summarise all above parameters for sake of simplicity. Intuitively, $T_{1}$ and $T_{2}$ account for the contributions of Rayleigh and Mie scatterings.

From Equation 4, we notice there is a linear relation between the non-scattered light $E_{s k y}(\lambda)$ and the sky light. This permits the use of a spectral ratio so as to describe the relation between the 
direct, i.e. non-scattered, light and the scattered skylight. The main advantage of this, as we will show later on, is that this ratio depends solely on the atmospheric conditions, such as humidity, cloud cover, etc. This dependence also hints at its usage in estimating overcast weather conditions.

\section{Scattering Spectral Ratio}

In this section, we commence by justifying the use of the scattering ratio in real-world lighting conditions making use of the spherical harmonic approach presented in [1]. Later on in this section, we present our scattering spectral ratio.

\subsection{Framework for General Lighting}

To deal with the settings in which the light strikes the object from every direction about the scene, Barsi and Jacobs[1] and Ramamoorthi and Hanrahan [28] have developed a theoretic framework based upon spherical harmonics.

Consider a Lambertian surface with bidirectional reflectance distribution (BRDF) $\rho\left(\omega_{i}, \omega_{o}\right)$, where $\omega_{i}$ corresponds to the incoming light direction and $\omega_{o}$ accounts for the outgoing light direction with respect to surface normal. The surface under consideration is illuminated by light impinging from all directions $\Omega=[0, \pi] \times[0,2 \pi]$. Here the light is expressed as $l\left(\theta_{l}, \phi_{l}\right)$, where $\left(\theta_{l}, \phi_{l}\right)$ denotes the light direction in the unit sphere. When the cast shadow and inter reflection is neglected, the intensity $I(u)$ at the pixel indexed $u$, with the surface normal $n$ is given as

$$
I(u)=\int_{\Omega} \rho\left(\omega_{i}, \omega_{o} ; n\right) l\left(\omega_{i}\right) \max \left(\cos \left(n, \omega_{i}\right), 0\right) d \omega_{i}
$$

The integration can be regarded as the convolution of the "spherical" lights with a kernel determined by the BRDF $\rho\left(\omega_{i}, \omega_{o}\right)$. Specifically, if the surface BRDF is Lambertian, the kernel will act as a low pass filter and, as noted by Basri and Jacobs [1], for any configuration of distant lighting, a second order spherical harmonics approximation will capture $98 \%$ of the reflectance. The intensity 
at pixel $u$ is, hence, given by

$$
I(u) \approx \Sigma_{i=1}^{K} l_{i} s_{i}
$$

where $K$ is chosen according to the order of the spherical harmonic selected, (e.g. 4 for the first order, 9 for the second order, etc. and $l_{i}$ and $s_{i}$ are the harmonic coefficients expanded from light function $l\left(\theta_{l}, \phi_{l}\right)$ and the half-cosine kernel for BRDF which "clamps" the negative values to zeros.

This implies that, if we view $l_{i}$ at each wavelength $\lambda$ as a product of the light power spectrum and its harmonic coefficient, we can conclude that the sunlight and skylight only depend on the direction and spatial distribution of the light. Similarly $s_{i}$ can be separated as the multiplication of surface albedo and the harmonic expansion $h_{i}$ for a kernel which only depends on the surface normal $n$. For a more detailed proof, please refer to Barsi and Jacob[1].

\subsection{Analysis on the shadow boundary}

Recall that, outdoor scenes are normally illuminated by two kinds of light, sunlight and skylight. Sunlight can be viewed as a point light source which, when occluded, produced a shadow in the scene. On the contrary, the skylight is a diffuse light which can be considered as uniformly spread across the scene.

Now let us consider the optic phenomenon on the shadow boundary. A pixel $u_{1}$ on the nonshadowed side of the shadow boundary is lit by both the sunlight and the skylight. The intensity of such a pixel can be written as

$$
\begin{aligned}
I_{\text {direct }}\left(\lambda, u_{1}\right) & =\sum_{i=1}^{K}\left(l_{i, \text { sun }}(\lambda)+l_{i, \text { sky }}\left(\lambda, u_{1}\right)\right) s_{i}\left(\lambda, u_{1}\right) \\
& =\sum_{i=1}^{K}\left(E_{\text {sun }}(\lambda) L_{i, \text { sun }}+E_{\text {sky }}(\lambda) L_{i, \text { sky }}\right) S\left(\lambda, u_{1}\right) h_{i}\left(u_{1}\right)
\end{aligned}
$$

where $S\left(\lambda, u_{1}\right)$ is the surface albedo, $l_{i, \text { sun }}(\lambda), l_{i, s k y}(\lambda)$ and $s_{i}\left(\lambda, u_{1}\right)$ are the harmonic coefficients akin to those in Equation 6, $E_{\text {sky }}(\lambda)$ and $E_{\text {sun }}(\lambda)$ are the power spectra of the sunlight and skylight 
at wavelength $\lambda$ and $L_{i, \text { sun }}$ and $L_{i, s k y}$ are the harmonic coefficients for the sunlight and skylight, respectively.

In the other hand, skylight, as mentioned earlier, can be considered as a diffuse light spread evenly across the scene. As the result, the nearby pixel $u_{2}$ on the shadowed side of the boundary can be viewed to have an intensity given by

$$
\begin{aligned}
I_{\text {shadow }}\left(\lambda, u_{2}\right) & =\sum_{i=1}^{K} l_{i, s k y}\left(\lambda, u_{2}\right) s_{i}\left(\lambda, u_{2}\right) \\
& =\sum_{i=1}^{K}\left(E_{\text {sky }}(\lambda) L_{i, s k y}\left(u_{2}\right)\right) S\left(\lambda, u_{2}\right) h_{i}\left(u_{2}\right)
\end{aligned}
$$

Since pixels $u_{1}$ and $u_{2}$ are close to each other, the difference between the two albedo $S\left(\lambda, u_{1}\right)$ and $S\left(\lambda, u_{2}\right)$ can be considered to be small. Moreover, the pixels on the two sides of the shadow boundary will often share the albedo due to being on the same object surface. Hence, we can write

$$
\frac{I_{\text {shadow }}(\lambda)}{I_{\text {direct }}(\lambda)-I_{\text {shadow }}(\lambda)}=\frac{S(\lambda) \sum_{i=1}^{K} E_{\text {sky }}(\lambda) L_{i, \text { sky }} h_{i}}{S(\lambda) \sum_{i=1}^{K} E_{\text {sun }}(\lambda) L_{i, \text { sun }} h_{i}}
$$

where we have used $S(\lambda)$ as an alternative to $S\left(\lambda, u_{2}\right)$ and $S\left(\lambda, u_{1}\right)$ and, for the sake of clarity, we have dropped the indices $u_{1}$ and $u_{2}$.

We can use the developments in the previous section and Equation 4 so as to write the expression above as follows

$$
\frac{I_{\text {shadow }}(\lambda)}{I_{\text {direct }}(\lambda)-I_{\text {shadow }}(\lambda)}=g\left(\frac{T_{1}}{\lambda^{4}}+\frac{T_{2}}{\lambda^{2}}\right)
$$

where $g=\frac{\sum_{i=1}^{K} L_{i, s k y} h_{i}}{\sum_{i=1}^{K} L_{i, \text { sun }} h_{i}}$ is wavelength independent coefficient which corresponds to the surface illumination geometry.

Note that the coefficient $g$ can be removed from further consideration via the normalisation of the ratio in Equation 10. This yields the Scattering Spectral Ratio (SSR)

$$
\varrho(\lambda)=\frac{\sum_{\lambda}\left(I_{\text {direct }}(\lambda)-I_{\text {shadow }}(\lambda)\right)}{\sum_{\lambda} I_{\text {shadow }}(\lambda)} \frac{I_{\text {shadow }}(\lambda)}{I_{\text {direct }}(\lambda)-I_{\text {shadow }}(\lambda)}
$$

The main advantage of the SSR is that this ratio only depends on the atmospheric conditions. This means that for the image under consideration all pixels on the shadow boundary are expected 
to share the same value of $\varrho(\lambda)$ regardless of the albedo, scene geometry or light power spectra. This opens up the possibility of finding the shadowed areas in the scene by recovering those boundaries across image regions subject to a consistent SSR over the image.

\section{Shaded Regions and Overcast Weather}

In this section, we turn our attention to the use of the SSR presented above for purposes of recovering the shaded regions in the image. We also ellaborate upon its application to the assessment of the overcast weather for the scene.

\subsection{Active Contours}

To separate the non-shaded areas in the image we can employ the fact that the SSR is a unique value across the shaded region boundaries. To do this, we make use of active contours. Active contours [2] are one of the most successful techniques in image segmentation. The basic idea is to evolve the contour for the boundary of the segmented region making use of constraints pertaining the problem in hand. Active contours can be categorised into two types. The first of these is based on an edge model, whereas the second group employs a region model. Here, we follow the developments in [37], where a formulation which combines the geodesic [4] and ChanVese [5] active contours formulations.

Here, we use the active contour model in [37] with a level set function which makes use of the ratio $\varrho(\lambda)$ to construct the region-based signed pressure force function for the shadowed areas in the image. This shadow force pressure function controls the evolution of the contour so as to encourage shrinkage of the delimited area about the sunlit regions and expansion across shadowed regions. To define our shadow force pressure function, we use the pixel-wise ratio $\varrho(\lambda)$ and note 
that this should equate to the value of

$$
\zeta\left(T_{1}, T_{2}, \lambda\right)=\left(\frac{T_{1}}{\lambda^{4}}+\frac{T_{2}}{\lambda^{2}}\right)
$$

yielded by $T_{1}$ and $T_{2}$ as global image parameters. This is as $T_{1}$ and $T_{2}$ account for both, the atmospheric conditions and the proportion of Rayleigh and Mie scatterings across the scene. Our shadow pressure function $F(u)$ is given by

$$
F(u)= \begin{cases}1 & \text { if }\left\|\varrho(\cdot)-\zeta\left(T_{1}, T_{2}, \cdot\right)\right\|>\varepsilon \\ -1 & \text { if }\left\|\varrho(\cdot)-\zeta\left(T_{1}, T_{2}, \cdot\right)\right\| \leq \varepsilon\end{cases}
$$

where $\varepsilon$ is a threshold value.

This yields the level set function of the form

$$
\frac{\partial \phi}{\partial t}=F(u) \beta\|\nabla(\phi)\|
$$

where $\beta$ is a parameter which governs the evolution speed of the contour.

To better understand the equations above, consider two sets of pixel-pairs on the image shown in the left-hand panel of Figure 1. The image is a hyperspectral one taken from the dataset in [11].
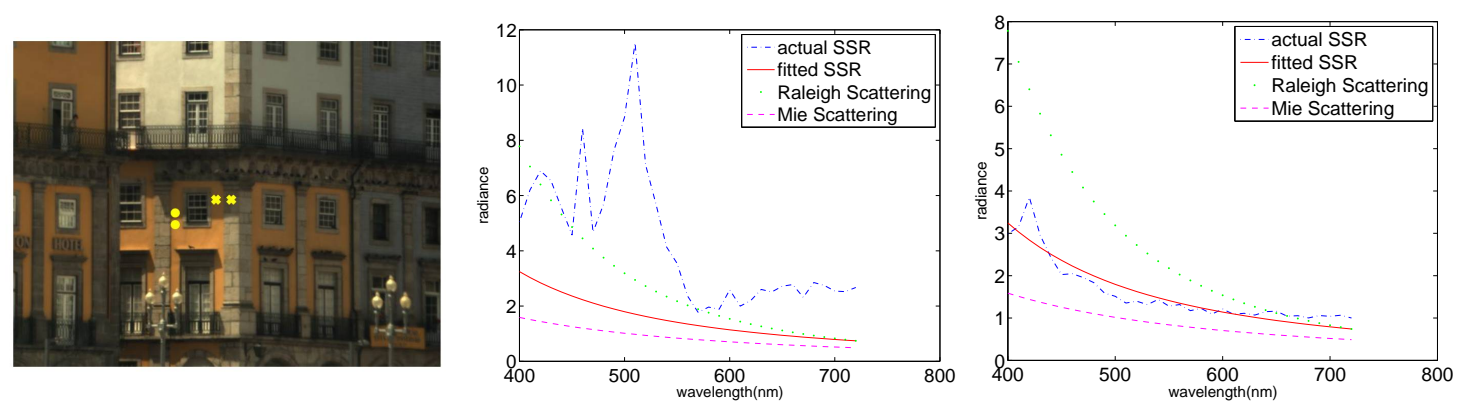

Figure 1: Spectral Scattering Ratio for two pixel-pairs taken from the sample image on the lefthand panel. The two panels on the right hand side show the SSR for a bogus and a real shadow boundary, respectively. The plots also show the predicted SSR (solid line) and the Mie (dashed line) and Rayleigh (dotted line) scattering. 
The two pixel-pairs, denoted on the image by crosses and circles, correspond to both, the case where the points lie on a bogus and real shadow boundary, respectively. We show the Scattering Spectral Ratio (SSR) for both pairs in the two right hand panels. Note that, for the bogus boundary, i.e. the plot in the middle panel, the expected SSR (solid line) is far removed from the actual Scattering Spectral Ratio (broken line). The figure also shows the expected contributions of the Mie and Rayleigh scattering in the dashed and dotted lines, respectively. In contrast, the shadow boundary SSR, plotted in the right-hand panel, closely fits the estimated Spectral Scattering Ratio.

\subsection{A Metric for Overcast Scenes}

With the shaded regions in hand, the ratio $\varrho(\lambda)$ can be used for purposes of inferring the amount to which the weather is overcast from a single image. This information can serve for environmental sciences, forensics and photography. Narasimhan and Nayar [25] estimate the weather condition from the glows of light sources in the atmosphere. This work only applies when some illuminants appears in the image. Photometric stereo has also been used to recover the global illumination to estimate weather as the light in a sunny day behaviours like a directional light [31]. This contrasts with overcast weather, where cloud acts as a diffuser. Lalonde, Efros and Narasimhan [20] propose a sun visibility cue to classify a set of features in a single image for purposes of light estimation. This work, despite effective, does not provide physical interpretation for this sun visibility cue.

Here, we note that the scattering spectral ratio (SSR), can be used to recover an overcast weather metric from a single image. Moreover, the SSR, with its footing on physics, provides a physical interpretation of the weather conditions in the scene. Note that, from Equation 11, the SSR on the shadowed region boundary is given by

$$
\varrho\left(\lambda_{c}\right)=\frac{\frac{T_{1}}{\lambda^{4}}+\frac{T_{2}}{\lambda^{2}}}{\sum_{c} \frac{T_{1}}{\lambda_{c}^{4}}+\frac{T_{2}}{\lambda_{c}^{2}}}
$$

where $c \in R, G, B$ is a sub-index that corresponds to the three colour channels. 
Note that, since the red and blue channels in a trichromatic image are not correlated (they do not overlap in the light wavelength domain), we are assured to have two independent values of $\varrho\left(\lambda_{c}\right)$, from which $T_{1}$ and $T_{2}$ can be estimated. Furthermore, since $T_{1}$ and $T_{2}$ are directly related to the turbidity, i.e. the contribution of the Mie and Rayleigh scatterings, they do account for the lighting conditions characteristic of the degree upon which the scene is overcast. This is as, in a clear sky without haze and the cloud, the value of $T_{1}$, i.e. the contribution of the Rayleigh scattering, should be high. In a sunny day $T_{2}$, which reflects the amount of Mie scattering, should be small. In the other hand, when the weather is overcast or hazy, $T_{1}$ will decrease while $T_{2}$ increases according to the proportion of Mie scattering in the scene. As a result, we can use the ratio

$$
\alpha=\frac{\frac{T_{1}}{T_{2}}-\alpha_{\text {overcast }}}{\alpha_{\text {clear }}-\alpha_{\text {overcast }}}
$$

in order to assess the weather conditions.

Note that, in the equation above, $\alpha$ can be viewed as the ratio $\frac{T_{1}}{T_{2}}$ scaled by $\alpha_{\text {overcast }}$ and normalised by the difference $\alpha_{\text {clear }}-\alpha_{\text {overcast }}$. We have done this so as to bound our metric between zero and unity by accounting for the difference of the ratio $\alpha$ between completely overcast and sunny conditions, i.e. $\alpha_{\text {overcast }}$ and $\alpha_{\text {clear }}$. In practice, we can estimate these two quantities empirically from a set of images, which, for the dataset used in our experiments, yielded

$$
\alpha=\frac{\frac{T_{1}}{T_{2}}-3 . \overline{33} \times 10^{4}}{5.66 \times 10^{4}}
$$

where $\alpha_{\text {overcast }}=3 . \overline{33} \times 10^{4}$ and $\alpha_{\text {clear }}=9 \times 10^{4}$.

\section{Implementation Issues}

In this section, we elaborate on the implementation issues regarding the estimation of the SSR through the recovery of shaded regions in the scene. Here, we present an iterative algorithm for the recovery of the shaded regions. We commence by describing the initialisation of the method. 
Later on, we ellaborate upon the updating procedure for the relevant parameters at each step of the algorithm.

\subsection{Initialisation}

We commence by noting that, following the developments presented earlier, we use $\zeta\left(T_{1}, T_{2}, \lambda\right)$ so as to compute the force pressure function $F(u)$ for the pixel $u$. This assumes that both parameters, $T_{1}$ and $T_{2}$ are available. In practice, this is generally not the case. In our implementation, we estimate the value of $\zeta\left(p_{c}, T_{1}, T_{2}\right)$ from the image itself.

To do this, we observe that the shadow boundaries should lie along the image edges. As a result, we use a Canny edge detector [3] so as to recover the edges in the image. In fact, our initialisation method is not overly sensitive to the selection of edge detector. Here, we have opted for the Canny edge detector so as to allow for a direct comparison with the method in [19]. Moreover, in outdoor scenes, as indicated in [20], objects normally stand up straight due to gravity. The shadow cast by these objects should have a long and straight boundary. Therefore, in our implementation, we choose to apply the detection algorithm in [18] so as to disregard short image edges. Once these edges are in hand, we search their neighborhood and treat the highest intensity pixel as a directly illuminated one, whereas the lowest intensity pixel is viewed as the shadowed one.

By assuming that the values of $\varrho(\lambda)$ for these edge pixels are normally distributed about $\zeta\left(p_{c}, T_{1}, T_{2}\right)$, we can use the expected value, i.e. their mean, as an estimator of $\zeta\left(p_{c}, \eta, \lambda\right)$. Thus, we have

$$
\bar{\zeta}(\lambda)=\frac{1}{|\Omega|} \sum_{\Omega} \varrho(\lambda)
$$

where $\Omega$ is the set of selected edge pixels in the image $\mathcal{I}$.

Note that, additionally to $\zeta\left(p_{c}, T_{1}, T_{2}\right)$, we are also required to initialise the shadow level set function. Here we divide the image into $M$ patches on a lattice and compute the ratio $\varrho_{c}$ (for the sake of consistency, we now use $c \in\{R, G, B\}$ as an alternative to the wavelength variable $\lambda$ ) 
for each pair of patches. We set the shadow level set function $\nabla(\phi)$ to unity at the patches where the ratio is in better accordance with the estimated $\bar{\zeta}_{k}$ for each of the colour channel. The shadow level set function is set to $\nabla(\phi)=-1$ everywhere else. At each iteration of the level-set evolution process for the active contour, we regularize the shadow level set function with a Gaussian filter $G_{\sigma}$ with zero-mean and standard deviation $\sigma$.

\subsection{Computing the Scattering Spectral Ratio}

Recall that, in Section 3, we used a single neighbour of the pixel $u$ to compute the corresponding ratio $\varrho(\lambda)$. To improve the robustness of the method to noise corruption and variations about the pixel $u$, we employ the ratio

$$
\varrho_{k}^{*}=\frac{1}{\ell(u)} \sum_{q=\{R, G, B\}} I_{q}(u) \frac{\left|\mathfrak{N}_{u}\right|}{\sum_{v \in \mathfrak{N}_{u}} I_{q}(v)}
$$

as an alternative to $\varrho(\lambda)$ in Equation 13.

In Equation 19, we have used $I_{k}(u)$ to denote the image brightness at pixel $u$ for the colour channel $k=\{R, G, B\}$, whereas $\ell(u)$ accounts for the pixel brightness and $\mathfrak{N}_{u}$ corresponds to the neighbourhood about the pixel $u$. We have done this since, in previous sections, we treated the problem in a general setting. This contrasts with our implementation, which aims at processing trichromatic imagery. Note that this can be done without any loss of generality since, for a surface with reflectance $S(\lambda)$ illuminated by a light source a power spectrum $E(\lambda)$, the value of a diffuse pixel $u$ follows the relation [36]

$$
I_{k}(u)=\int_{\Lambda_{k}} E(\lambda) S(\lambda) R(\lambda) d \lambda
$$

where $R(\lambda)$ denotes the camera's colour sensitivity function over the operating wavelength range $\Lambda_{k}$ for each of the colour channels $k \in\{R, G, B\}$. This linear relation, compounded by the fact that we can recover $\zeta\left(p_{c}, \eta, \lambda\right)$ devoid of the reflectance making use of the pixel values allows for 
a similar treatment of Equation 18, where the colour channel index can be used as an alternative to the wavelength $\lambda$.

\subsection{Updating the Force Pressure Function}

Note that for the initialisation in Section 5.1, the $\zeta\left(T_{1}, T_{2}, \lambda\right)$ is estimated from the edges of the image. This is used as an initial guess. At each subsequent iteration, we re-estimate the value of $\zeta(\cdot)$ from the current shadow boundaries. This updates continue until convergence is reached.

For each iteration, as the shadow level set evolves, the shadow boundary is extracted. In practice, this is done by applying a morphological dilation on the level set function so as to expand it. The level set function is shrunk by applying morphological erosion. After deducing the eroded image from the dilated version, the boundary comes out.

We neglect the boundary pixels which are close to the boundary of the image. Following section 5.1, this is only applied to long boundaries. In our implementation, if a pixel lies on the a boundary for three iterations, it is treated as a true shadow boundary pixel. The algorithm is considered to have converged once no more updates are effected upon the boundaries in the image

At each iteration, the scattering spectral ratio is computed as follows. Recall that, from Equation 18 , the SSR for the pixels is solely governed by $T_{1}$ and $T_{2}$. This can be expressed in matrix form as

$$
\left[\begin{array}{cc}
\varrho_{1}\left(\lambda_{1}\right) \sum_{k} \frac{1}{\lambda_{k}^{4}}-\frac{1}{\lambda_{1}^{4}} & \varrho_{1}\left(\lambda_{1}\right) \sum_{c} \frac{1}{\lambda_{k}^{2}}-\frac{1}{\lambda_{1}^{2}} \\
\varrho_{1}\left(\lambda_{2}\right) \sum_{k} \frac{1}{\lambda_{k}^{4}}-\frac{1}{\lambda_{2}^{4}} & \varrho_{1}\left(\lambda_{2}\right) \sum_{c} \frac{1}{\lambda_{k}^{2}}-\frac{1}{\lambda_{2}^{2}} \\
\varrho_{2}\left(\lambda_{1}\right) \sum_{k} \frac{1}{\lambda_{k}^{4}}-\frac{1}{\lambda_{1}^{4}} & \varrho_{1}\left(\lambda_{1}\right) \sum_{c} \frac{1}{\lambda_{k}^{2}}-\frac{1}{\lambda_{1}^{2}} \\
\varrho_{2}\left(\lambda_{2}\right) \sum_{k} \frac{1}{\lambda_{k}^{4}}-\frac{1}{\lambda_{2}^{4}} & \varrho_{2}\left(\lambda_{2}\right) \sum_{c} \frac{1}{\lambda_{k}^{2}}-\frac{1}{\lambda_{2}^{2}} \\
\vdots & \vdots \\
\varrho_{M}\left(\lambda_{1}\right) \sum_{c} \frac{1}{\lambda_{k}^{4}}-\frac{1}{\lambda_{1}^{4}} & \varrho_{M}\left(\lambda_{1}\right) \sum_{c} \frac{1}{\lambda_{k}^{2}}-\frac{1}{\lambda_{1}^{2}} \\
\varrho_{M}\left(\lambda_{2}\right) \sum_{c} \frac{1}{\lambda_{k}^{4}}-\frac{1}{\lambda_{2}^{4}} & \varrho_{M}\left(\lambda_{2}\right) \sum_{c} \frac{1}{\lambda_{k}^{2}}-\frac{1}{\lambda_{2}^{2}}
\end{array}\right]\left[\begin{array}{c}
0 \\
T_{1} \\
T_{2}
\end{array}\right]=\left[\begin{array}{c}
0 \\
0 \\
0 \\
\vdots \\
0 \\
0
\end{array}\right]
$$


where $\varrho_{i}(\lambda)$ corresponds to the SSR value for $\lambda$ wavelength of $i_{t h}$ pixel on the shadow boundary.

As discussed above, for a trichomatic image, we employ the red and the blue channels for the computation of $T_{1}$ and $T_{2}$. Using the equation above, we recover $T_{1}$ and $T_{2}$ via linear least squares and make use of Equation 12 so as to update $\zeta\left(T_{1}, T_{2}, \lambda\right)$ for the next iteration.

\subsection{Estimating the Penumbra}

Despite being effective, Active Contours are prone to failure in areas where the illuminance is a mixture of close to equal contributions of sunlight and skylight, i.e. regions of penumbra. In order to address this drawback, we employ the KNN matting method proposed by Chen et al.[6]. Note that the KNN matting method hinges in the use of the k-nearest neighbours about the pixel under consideration to achieve a closed form solution to the problem of image matting.

Since penumbra normally ensues on the transitions between shadowed and non-shadow regions in the image, we apply the KNN matting method to the detected boundaries. This is done after our method method converges. To recover the shadow-sunlit samples, we employ a series of morphological erosion operations to both, the shadow and sunlit regions. This effectively recovers the central pixels for the recovered regions while, at the same time, removes small spurious regions.

\section{Experiments}

In this section we illustrate the utility of our method for purposes of shadow detection. To this end, we use a set of real-world images captured in the outdoors under a variety of atmospheric conditions making use of a Nikon D80. These vary from images with a large amount of cloud cover and high humidity to scenes with no clouds. In all our experiments, we have set the number $M$ of patches used for the initialisation of the level set function to $25 \times 25$, the neighbourhood $\mathfrak{N}_{u}$ is given by $15 \times 15$ pixels, the threshold $\epsilon$ is set to 0.3 , the standard deviation of the Gaussian filter 
is $\sigma=1$ and $\beta$ is set as 25 .

As the dataset is comprised of 12 photos in different weather conditions. For our experiments, we separate them into three subsets according to the weather at the time of acquisition. The photos are hence separated into "clear day", "half cast" and "overcast" subsets. The imagery in the "clear day" subset are those taken in a sunny day without any cloud on the sky. The images in the half cast subset were taken when the sky was not completely overcast nor the day was outright sunny. The overcast subset contains images taken when the sky was totally covered by cloud.

Here we commence by show shadow detection results on these three subset of images. We then explore the utility of the ratio $\alpha$ for purposes of overcast weather estimation. Finally, we show results on time complexity.

\subsection{Shadow Detection}

For our shadow detection experiments, we provide comparison with two alternatives. These are the methods in [19] and the algorithm presented in [14]. The method in [19] employs a decision tree classifier whereas the algorithm in [14] is a region-based approach that performs pairwise classification so as to achieve shadow detection.

In Figure 2, we show the detection results for our algorithm and the alternatives when applied to sample images in our dataset. In the figures, the left-hand column shows the detection results yielded by our method whereas the middle and right-hand column show the result from the methods in [19] and [14], respectively. From Figure 2, we can conclude that our method provides a margin of improvement over the alternatives. Note that, with the exception of the two shadows corresponding to the two tree canopies in the images on the second and fourth columns, our method successfully recovers the shadow boundaries. These failures are due to the fact that the boundary recovered by our method is consistent with the scattering theory used here. Since these canopies act as diffusers, these shadows are rather a mix of sunlight and skylight. Moreover, our method 


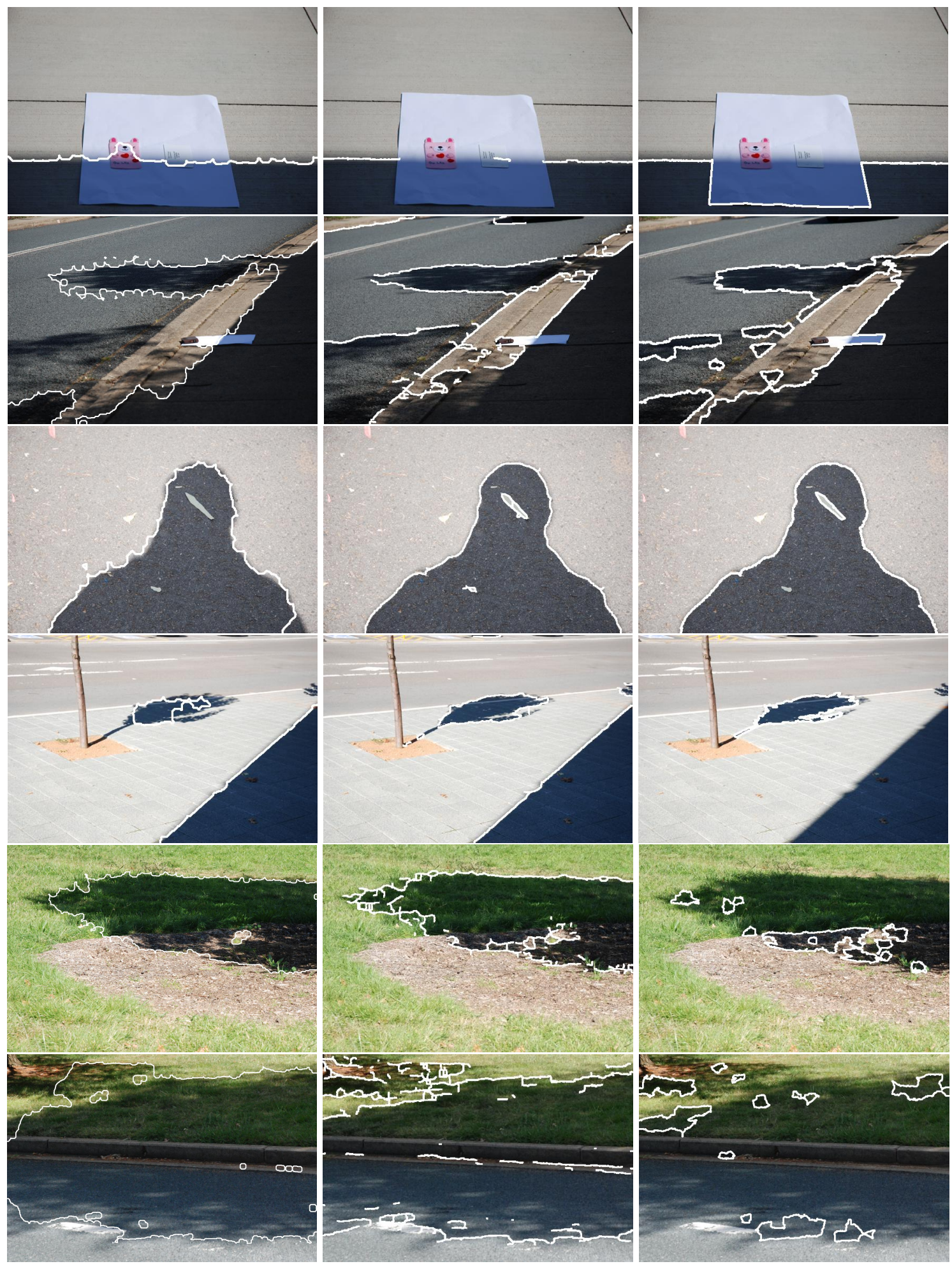

Figure 2: Shadow detection results for our method and the alternatives. From left-to-right: The regions recovered by our method, those yielded by the method in [19] and the shadows recovered by the approach in [14] 
performs particularly well on the image in the first row, where no other method can recover the shadows over the white paper. This is also evident in the third row, where the leaf in the middle of the shadow is classified by the methods in [19] and [14] as non-shadowed regions. Moreover, despite effective, the method in [19] does not necessarily deliver a closed shadow boundary. Note that this can potentially hinder shadow removal methods hinging in region filling or matting approaches, such as that in [35].

Now we turn our attention to the alpha mattes recovered via the application of KNN matting. In Figure 3, we show the input images, shadow and sunlit layers in the left-hand, middle and right-hand columns, respectively. From the results, we can conclude that the recovered layers do follow the natural transitions from shadow to sunlit regions in the imagery. This can be seen in the shadows for buildings, where the extracted layer shifts from shadow to sunlit is noticeably sharper than those for the tree canopies with a more gradual transition between layers. Thus, by making use of matting as a post-processing step, our method can cope with cases where there is no sharp boundary between shadows and sunlit regions. These are, in general not well suited for the active contours since they violate the binary classification assumption for sharp shadow boundaries.

\subsection{Performance for Weather Estimation}

We now turn our attention to the value of $\alpha$. Following Section 4.2, we proceed to estimate the degree upon which the weather is overcast from the boundary information for each image. To this end, we have separated our imagery into three subsets corresponding to clear sky, half cast and overcast scenes. In Fig 4 we show sample images for each of these image subsets. We have then proceeded to compute the mean and standard deviation of $\alpha$ for the imagery corresponding to each of these three weather conditions.

Table 1 shows the mean value and standard deviation of $\alpha$ for the three subsets. From the table, it is evident that $\alpha$ does reflect the weather condition. Note that the general trend is, as expected, for 

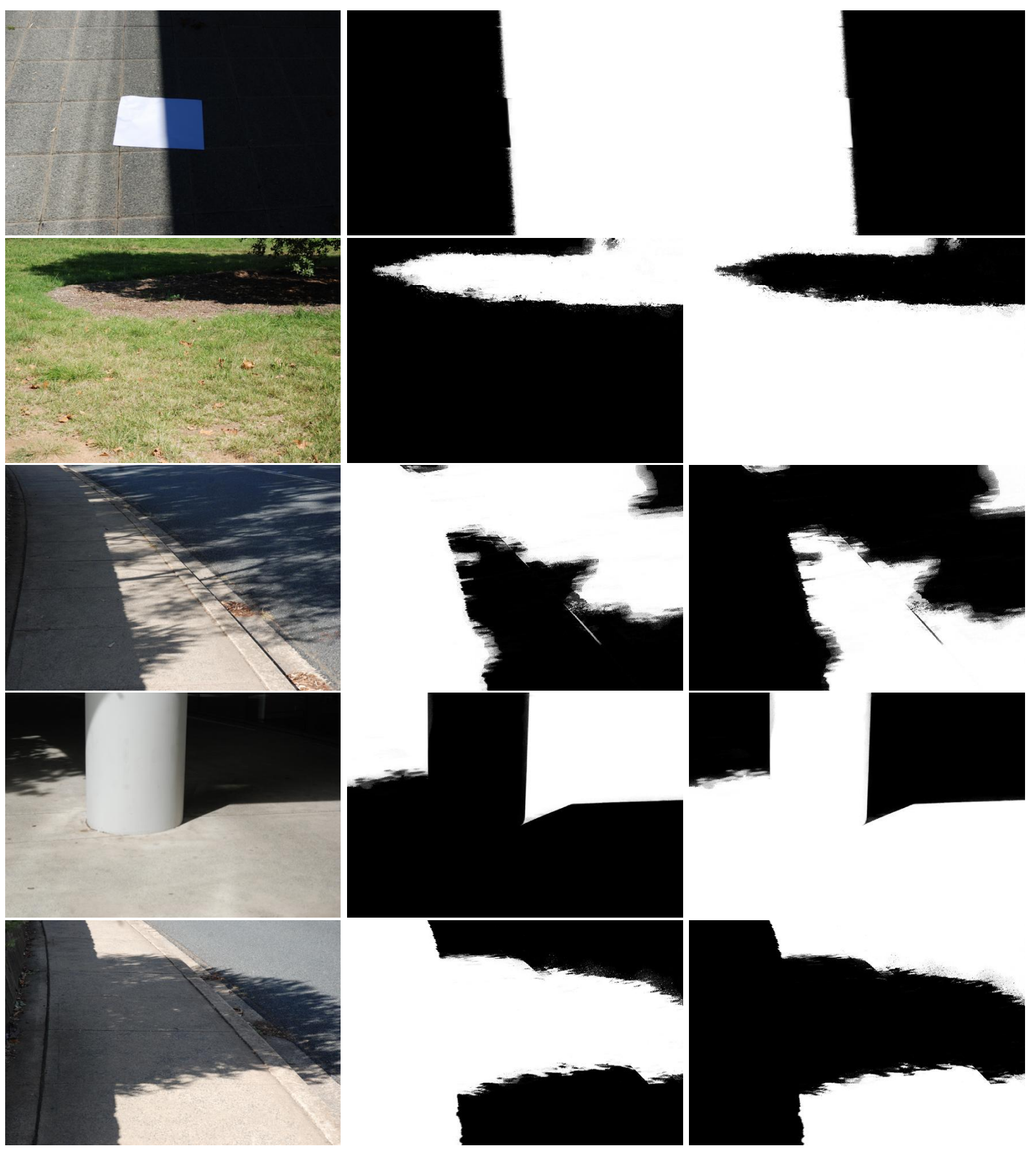

Figure 3: Shadow and sunlit area layers recovered via the application of KNN matting. From left to right, we show the input image, the shadow and sunlit area layers, respectively.

$\alpha$ to decrease with the amount of cloud cover. This is in accordance with the physical interpretation of $\alpha$ as discussed earlier. 

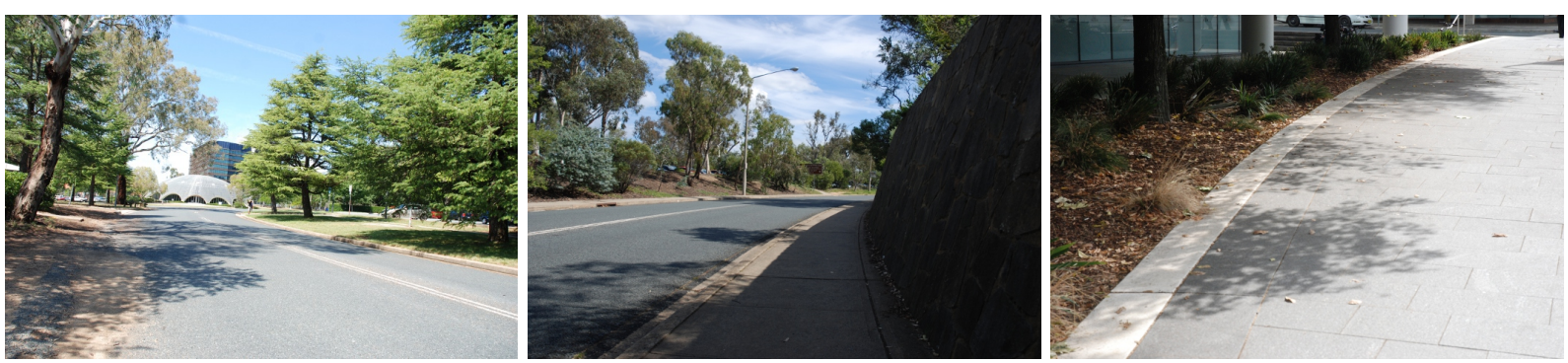

Figure 4: Samples of three subsets, the images from left to right belong to clear day, half cast and overcast subset respectively

Table 1: Value of $\alpha$ for the three image subsets used in our experiments.

\begin{tabular}{|l|c|r|}
\hline Clear sky & Half cast & Overcast \\
\hline $0.8708 \pm 0.6489$ & $0.7147 \pm 0.5275$ & $0.3352 \pm 0.8271$ \\
\hline
\end{tabular}

\subsection{Time Complexity}

Finally, we examine the time complexity of our method as compared with the alternatives. All the methods under consideration were implemented on a Window XP work station with an Intel Quad Core CPU at 2.66GHz. The average time per-image in our dataset for each method is reported in the Table 1. All the results are reported in seconds.

From the table, we can see that our method is more efficient than the alternatives. Indeed, it is almost 10 times faster than the method [14]. Note that, although it is only slightly faster than the method proposed by Lalonde et al. [19], the alternative is only aimed at recovering the shadow boundary. This contrasts with our method, which recovers the shadowed regions across the image.

Table 2: Execution times for our method and the alternatives. The table shows the mean processing time and the corresponding standard deviation (in seconds) per image in our dataset.

\begin{tabular}{|l|l|r|}
\hline Our Method & Method in [14] & Approach in [19] \\
\hline $156.3 \pm 125.8$ & $1354.0 \pm 3563.7$ & $184.7 \pm 62.0$ \\
\hline
\end{tabular}




\section{Conclusions}

In this paper, we have presented a shadow modelling approach which hinges in the use of Rayleigh scattering and Mie theory. This allows for the detection of shadows in outdoor imagery based upon a scattering ratio which reflects the proportion of skylight and sunlight across the scene. For the recovery of shadowed regions, this ratio is used to govern the evolution of an active contour across the image. This allows for the shadow detection problem to be treated as a segmentation one. We have also used the spectral ratio for purposes of defining a metric which can be used to assess the degree upon which the scene is overcast. We have provided results on real-world imagery and compared our method against alternatives elsewhere in the literature.

\section{References}

[1] R. Basri and D. W. Jacobs. Lambertian reflectance and linear subspaces. IEEE Transactions on Pattern Analysis and Machine Intelligence, , 25(2):218-233, 2003.

[2] A. Blake and M. Isard. Active Contours: The Application of Techniques from Graphics, Vision, Control Theory and Statistics to Visual Tracking of Shapes in Motion. SpringerVerlag, 1998.

[3] J. Canny. A computational approach to edge detection. IEEE Transactions on Pattern Analysis and Machine Intelligence, 8(6):679-698, 1986.

[4] V. Caselles, R. Kimmel, and G. Sapiro. Geodesic active contours. In International Conference on Computer Vision, pages 694-699, 1995.

[5] T. F. Chan and L. A. Vese. Active contours without edges. IEEE Transactions on Image Processing, 10(2):266-277, 2001. 
[6] Q. Chen, D. Li, and C. Tang. KNN matting. IEEE Transactions on Pattern Analysis and Machine Intelligence, 35(9):2175-2188, 2013.

[7] CIE. Spatial distribution of daylight - CIE standard general sky. Technical report, The International Commission on Illumination.

[8] R. Cucchiara, C. Grana, M. Piccardi, and A. Prati. Detecting moving objects, ghosts, and shadows in video streams. IEEE Transactions on Pattern Analysis and Machine Intelligence, 25(10):1337-1342, 2003.

[9] L. Z. Fang, W. Y. Qiong, and Y. Z. Sheng. A method to segment moving vehicle cast shadow based on wavelet transform. Pattern Recognition Letters, 29(16):2182-2188, 2008.

[10] G. Finlayson, M. Drew, and C. Lu. Entropy minimization for shadow removal. International Journal of Computer Vision, 85(1):35-57, 2009.

[11] D. Foster, K. Amano, S. M. C. Nascimento, and M. Foster. Frequency of metamerism in natural scenes. J. Opt. Soc. Am. A, 23(10):2359-2372, Oct 2006.

[12] G. Funka-Lea and R. Bajcsy. Combining color and geometry for the active, visual recognition of shadows. In International Conference on Computer Vision, pages 203-209, 1995.

[13] L. Gu and A. Robles-Kelly. Shadow detection via rayleigh scattering and mie theory. In International Conference on Pattern Recognition, pages 2165-2168, 2012.

[14] R. Guo, Q. Dai, and D. Hoiem. Single-image shadow detection and removal using paired regions. In Conference on Computer Vision and Pattern Recognition, 2011.

[15] J. W. Hsieh, W. F. Hu, C. J. Chang, and Y. S. Chen. Shadow elimination for effective moving object detection by gaussian shadow modeling. Image and Vision Computing, 21(6):505-516, 2003. 
[16] M. Kerker. The scattering of light, and other electromagnetic radiation. Academic Press, 1969.

[17] D. C. Knill, D. Kersten, and P. Mamassian. Geometry of shadows. J. Opt. Soc. Amer. A, 14(12):3216-3232, 1997.

[18] J. Kosecka and W. Zhang. Video compass. In European Conference on Computer Vision, pages 476-490. 2002.

[19] J. Lalonde, A. Efros, and S. Narasimhan. Detecting ground shadows in outdoor consumer photographs. In European Conference on Computer Vision, 2010.

[20] J. Lalonde, A. A. Efros, and S. G. Narasimhan. Estimating the natural illumination conditions from a single outdoor image. International Journal of Computer Vision, 98(2):123-145, 2012.

[21] A. Leone and C. Distante. Shadow detection for moving objects based on texture analysis. Pattern Recognition, 40(4):1222-1233, 2007.

[22] N. Martel-Brisson and A. Zaccarin. Moving cast shadow detection from a gaussian mixture shadow model. In Computer Vision and Pattern Recognition, pages 643-648, 2005.

[23] M. Minnaert. Light and Color in the Open Air. Dover, 1954.

[24] S. G. Narasimhan and S. K. Nayar. Vision and the atmosphere. International Journal of Computer Vision, 48(3):233-254, 2002.

[25] S. G. Narasimhan and S. K. Nayar. Shedding light on the weather. In Computer Vision and Pattern Recognition, pages 665-672, 2003.

[26] F. Porikli and J. Thornton. Shadow flow: A recursive method to learn moving cast shadows. In International Conference on Computer Vision, pages 891-898, 2005. 
[27] A. J. Preetham, P. Shirley, and B. Smits. A practical analytic model for daylight. In Proceedings of the 26th annual conference on Computer graphics and interactive techniques, SIGGRAPH '99, pages 91-100, 1999.

[28] R. Ramamoorthi and P. Hanrahan. A signal-processing framework for inverse rendering. In Proceedings of the 28th annual conference on Computer graphics and interactive techniques, SIGGRAPH '01, pages 117-128, 2001.

[29] E. Salvador, A. Cavallaro, and T. Ebrahimi. Cast shadow segmentation using invariant color features. Computer Vision and Image Understanding, 95(2):238-259, 2004.

[30] A. Sanin, C. Sanderson, and B. C. Lovell. Improved shadow removal for robust person tracking in surveillance scenarios. In International Conference on Pattern Recognition, pages $141-144,2010$.

[31] L. Shen and P. Tan. Photometric stereo and weather estimation using internet images. In Computer Vision and Pattern Recognition, pages 1850-1857, 2009.

[32] J. Tian, J. Sun, and Y. Tang. Tricolor attenuation model for shadow detection. IEEE Transactions on Image Processing, 18(10):2355-2363, 2009.

[33] Y. Wang, K. F. Loe, and J. K. Wu. A dynamic conditional random field model for foreground and shadow segmentation. IEEE Transactions on Pattern Analysis and Machine Intelligence, 28(2):279-289, 2006.

[34] T. P. Wu and C. K. Tang. A bayesian approach for shadow extraction from a single image. In International Conference on Computer Vision, pages 480-487, 2005.

[35] T. P. Wu, C. K. Tang, M. S. Brown, and H. Y. Shum. Natural shadow matting. ACM Transactions on Graphics, 26(2), 2007. 
[36] G. Wyszecki and W.S. Stiles. Color Science: Concepts and Methods, Quantitative Data and Formulae. Wiley, 2000.

[37] K. Zhang, L. Zhang, H. Song, and W. Zhou. Active contours with selective local or global segmentation: A new formulation and level set method. Image and Vision Computing, 28(4):668-676, 2010. 